\title{
Recommendations for returning genomic incidental findings? We need to talk!
}

\author{
Wylie Burke, MD, PhD ${ }^{1}$, Armand H. Matheny Antommaria, MD, PhD², \\ Robin Bennett, MS, $\mathrm{CGC}^{3}$, Jeffrey Botkin, MD, $\mathrm{MPH}^{4}$, Ellen Wright Clayton, MD, JD ${ }^{5}$, \\ Gail E. Henderson, $\mathrm{PhD}^{6}$, Ingrid A. Holm, MD, $\mathrm{MPH}^{7-9}$, Gail P. Jarvik, MD, PhD, \\ Muin J. Khoury, MD, PhD ${ }^{10}$, Bartha Maria Knoppers, JD, PhD ${ }^{11}$, Nancy A. Press, PhD ${ }^{12}$, \\ Lainie Friedman Ross, MD, PhD ${ }^{13}$, Mark A. Rothstein, JD ${ }^{14}$, Howard Saal, MD ${ }^{15}$, \\ Wendy R. UhImann, MS, CGC ${ }^{16}$, Benjamin Wilfond, MD ${ }^{17}$, Susan M. Wolf, JD ${ }^{18}$ \\ and Ron Zimmern, FRCP, FFPHM ${ }^{19}$
}

The American College of Medical Genetics and Genomics recently issued recommendations for reporting incidental findings from clinical whole-genome sequencing and whole-exome sequencing. The recommendations call for evaluating a specific set of genes as part of all whole-genome sequencing/whole-exome sequencing and reporting all pathogenic variants irrespective of patient age. The genes are associated with highly penetrant disorders for which treatment or prevention is available. The effort to generate a list of genes with actionable findings is commendable, but the recommendations raise several concerns. They constitute a call for opportunistic screening, through intentional effort to identify pathogenic variants in specified genes unrelated to the clinical concern that prompted testing. Yet for most of the genes, we lack evidence about

The American College of Medical Genetics and Genomics (ACMG) recently issued recommendations for reporting of incidental findings when whole-genome sequencing (WGS) or whole-exome sequencing (WES) is used in clinical practice. ${ }^{1}$ Like most guidelines, these recommendations are intended to promote a standard of practice. The article includes a disclaimer that notes "It may be prudent ... to document in the patient's record the rationale for any significant deviation from these recommendations." ' Yet genome sequencing represents an emerging technology for which there is little accumulated clinical experience or evidence. Many questions remain about its analytic performance and optimal use, and outcome data are largely lacking. Furthermore, the ACMG recommendations challenge accepted norms of patient autonomy and testing, the predictive value of testing, genotype penetrance, spectrum of phenotypes, and efficacy of interventions in unselected populations. Furthermore, the recommendations do not allow patients to decline the additional findings, a position inconsistent with established norms. Finally, the recommendation to return adult-onset disease findings when children are tested is inconsistent with current professional consensus, including other policy statements of the American College of Medical Genetics and Genomics. Instead of premature practice recommendations, we call for robust dialogue among stakeholders to define a pathway to normatively sound, evidence-based guidelines.

Genet Med advance online publication 1 August 2013 especially in children. In this article, we review four substantive concerns raised by the ACMG recommendations and consider the related issues that should be addressed in constructive dialogue among stakeholders, with the goal of defining a pathway to normatively sound, evidence-based guidelines that can be broadly supported.

\section{ESSENTIAL ELEMENTS OF THE ACMG RECOMMENDATIONS}

The ACMG statement recommends that when WGS or WES is performed for any clinical purpose in children or adults, a specified list of 56 genes associated with 24 inherited conditions be assessed, in addition to the genomic information for which testing was sought. The conditions were selected on the

\footnotetext{
${ }^{1}$ Department of Bioethics and Humanities, University of Washington, Seattle, Washington, USA; ${ }^{2}$ Ethics Center, Cincinnati Children's Hospital Medical Center, Cincinnati, Ohio, USA; ${ }^{3}$ Division of Medical Genetics, Department of Medicine, University of Washington, Seattle, Washington, USA; ${ }^{4}$ Department of Pediatrics, University of Utah, Salt Lake City, Utah, USA; ${ }^{5}$ Center for Biomedical Ethics and Society and School of Law, Vanderbilt University, Nashville, Tennessee, USA; ${ }^{6}$ Department of Social Medicine, University of North Carolina at Chapel Hill, Chapel Hill, North Carolina, USA; ${ }^{7}$ Division of Genetics, Boston Children's Hospital, Boston, Massachusetts, USA; ${ }^{8}$ The Manton Center for Orphan Disease Research, Boston Children's Hospital, Boston, Massachusetts, USA; ${ }^{9}$ Department of Pediatrics, Harvard Medical School, Boston, Massachusetts, USA; ${ }^{10}$ Office of Public Health Genomics, Centers for Disease Control and Prevention, Atlanta, Georgia, USA; ${ }^{11}$ Centre of Genomics and Policy, McGill University, Montreal, Quebec, Canada; ${ }^{12}$ Schools of Nursing and Medicine, Oregon Health and Science University, Portland, Oregon, USA; ${ }^{13}$ Department of Pediatrics and the MacLean Center for Clinical Medical Ethics, University of Chicago, Chicago, Illinois, USA; ${ }^{14}$ Institute for Bioethics, Health Policy and Law, University of Louisville School of Medicine, Louisville, Kentucky, USA; ${ }^{15}$ Division of Human Genetics, Cincinnati Children's Hospital Medical Center, Cincinnati, Ohio, USA; ${ }^{16}$ Division of Molecular Medicine and Genetics, Department of Internal Medicine and Department of Human Genetics, University of Michigan, Ann Arbor, Michigan, USA; ${ }^{17}$ Treuman Katz Center for Pediatric Bioethics, Seattle Children's Research Institute and Department of Pediatrics, University of Washington, Seattle, Washington, USA; ${ }^{18}$ Law School, Medical School, and Center for Bioethics, University of Minnesota, Minneapolis, Minnesota, USA; ${ }^{19}$ Foundation for Genomics and Population Health, Cambridge, UK. Correspondence: Wylie Burke (wburke@u.washington.edu)
} 
basis of high penetrance and clinical actionability. Variants to be reported from this assessment include those known and predicted to be pathogenic. The recommendations specify that these results, which are considered incidental findings, should be generated by the laboratory and reported regardless of the age of the patient. The recommendations reject the idea that the patient should be able to refuse these findings, as the authors state that they "did not favor offering the patient a preference as to whether or not their clinician should receive a positive finding from the minimum list of incidental findings described in these recommendations. We recognize that this may be seen to violate existing ethical norms regarding the patient's autonomy and "right not to know" genetic risk information".

\section{INCIDENTAL FINDINGS?}

Incidental findings are frequently identified in the course of clinical practice. They derive from the evaluation done to answer the clinical question and can range from unanticipated skin findings on a physical examination to an unexplained mass on a radiological study. When they occur, clinicians must consider their clinical significance and the appropriate information to offer patients about them.

The evaluation of the 56 genes called for in the ACMG recommendations do not constitute incidental findings in the usual sense of the term: they are more specific and intentional. The recommendations call for purposeful analytic effort, above and beyond what is required to answer the clinical question that prompted WGS/WES. If the 56 extra genes were not analyzed, no finding(s) would be available to report, and the results of the extra analysis represent information not sought by the patient.

At issue is not simply a debate about the application of "incidental findings" terminology to analysis of the 56 genes, but more important, the implications of this added analysis for clinicians and their patients. In emphasizing a specific list of genes to evaluate routinely as part of WGS/WES, the recommendations endorse the decision not to interrogate other genes. Among those other genes are some associated with highly penetrant conditions that currently lack medical treatment, such as early-onset Alzheimer disease, and some associated with conditions of low penetrance that are highly actionable, such as HFE-associated hemochromatosis. In short, the list proposed in the ACMG recommendations is a highly selected subset of the findings potentially obtainable from WGS/WES.

A selective approach to analysis of WGS/WES is both wise and necessary. Over time, interrogation of DNA sequence will likely become increasingly automated, but the process of whole-genome or whole-exome analysis will remain selective for the foreseeable future. Attempting a full analysis of all 20,000 genes and clinically significant noncoding regions is neither feasible in clinical care nor advisable, given variability in clinical significance and difficulty in interpretation of clinical implications for much of the genome. Laboratories and clinicians therefore choose to analyze certain portions of the genome and not others. In this context, assessment of the 56-gene panel represents a distinct addition to the analytic process, intended to achieve a specific purpose unrelated to the reason the test was ordered.

The ACMG recommendations suggest that once the sequence is in hand, "it becomes relatively easy for a laboratory to report a limited number of variants for conditions that could be medically important,"' but evaluation of DNA sequence to identify pathogenic variants is in fact labor intensive. Many findings represent rare or novel changes, and current databases and scientific literature include errors and uncertainty. For example, Jarvik et al. ${ }^{2}$ recently reported an expert review of 1,000 research participants' exomes for variants in 118 actionable genes. They reviewed 239 unique variants reported to be "disease causing" by the Human Genome Mutation Database. On review of the primary literature, which took more than 92 hours of expert time, only 9 variants in 8 participants met criteria for a pathogenic mutation. These included 7 variants in genes on the ACMG list. This calculation does not fully capture the effort involved in assessing variants. Jarvik et $\mathrm{al}^{2}{ }^{2}$ gained efficiency by concurrent assessment of results from 1,000 subjects; these 239 variants were found 585 times in the sample. If $50 \%$ of exomes have variants that require evaluation, and these evaluations require $>20$ min each, excluding double review and locating relevant literature, considerable time can be invested in the process of variant review for the discovery of remarkably few diseasepredisposing variants.

The ACMG statement invokes the analogy of medical imaging as a related technology that generates findings unrelated to the clinical question at hand. ${ }^{1}$ When a clinician orders a magnetic resonance imaging study of the chest to evaluate a mass, for example, the radiologist may note additional findings in the heart or lungs of potential clinical significance, which will be included in the imaging report. Despite surface similarities, this analogy is not entirely applicable to genome sequencing. The analysis used to generate the clinically relevant magnetic resonance imaging result also generates the incidental finding, in that the radiologist cannot avoid detecting the finding in the course of completing the analysis required to address the clinical question. By contrast, in WGS/WES, the genes relevant to the patient's clinical presentation can be analyzed without including the 56-gene panel (unless one or more of those genes is directly relevant to the patient's clinical presentation). In other words, the ACMG recommendations call for a deliberate add-on to the analytic effort, focusing on evaluation of a small subset of genes deemed by the ACMG to offer high potential for patient benefit.

It is not surprising that a technology as innovative as WGS/ WES lacks an exact analogy in existing tests. Recognition of the deliberate decisions involved in generating ancillary or secondary findings from this testing process can be helpful in developing appropriate testing policies. In doing so, we suggest the need for further attention to limitations in current evidence and incorporation of patient choice into the WGS/WES testing process. 


\section{EVIDENCE}

The authors of the ACMG recommendations acknowledge that "there are insufficient data on clinical utility to fully support these recommendations,"1 and accordingly encourage periodic revisiting of their list. The recommendations are not accompanied by a technical report or systematic review. However, the underlying rationale for the selection of the genes is clear. The recommendations focus on inherited mutations in genes associated with highly penetrant diseases for which interventions are available either to ameliorate the course of the disease or to provide prevention opportunities. The current standard of practice in medical genetics would call for offering testing to any patient with symptoms suggesting these conditions.

Although the intent of the recommendations is commendable - to find treatable disease before it is symptomatic-the lack of evidence for this use of genome sequencing suggests that practice recommendations are premature. WGS/WES is currently done in a few well-defined clinical circumstances: ${ }^{1,3}$ for patients with suspected genetic conditions for which conventional genetic testing has failed to provide a diagnosis or for which a specific genetic test is not available; as a tool for assessing genetically heterogeneous conditions; in cases in which comparison of the patient's genome with a tumor genome may offer a guide to therapy; and in cases in which a genetic predisposition may exist to a rare cancer or group of cancers. In all of these situations, a patient has a clinical condition or family history that points to a specific clinical concern that the testing can address. With respect to the 56 additional genes to be routinely assessed, however, most patients undergoing WGS/WES will be asymptomatic; nothing about their clinical presentation will necessarily point to the need to assess these genes.

The potential to identify treatable or preventable genetic diseases at an early stage, in order to improve outcome, is promising. ${ }^{4}$ However, the approach stipulated in the ACMG recommendations represents a form of opportunistic screening - not unlike checking a person's blood pressure when he or she seeks care for a respiratory infection. In other words, evaluation of the 56-gene panel has a different purpose from the analysis of sequence data being done to address the patient's presenting complaint. There are substantive concerns about this use of sequencing data. Many of the 56 genes have only recently been identified, and few of the disorders have a well-defined natural history. Mutations identified as pathogenic have been seen for the most part in patients who presented with the disease, leading to concerns about ascertainment bias. ${ }^{5,6}$ As a result, there is uncertainty about the full spectrum of clinical manifestations associated with the mutations and about the penetrance of risk genotypes among unselected patients. There is also a general lack of controlled studies of interventions.

It is an established precept of public health that screening should be instituted only when there is compelling evidence that it improves health outcomes in asymptomatic people. ${ }^{7}$ The value of finding and treating hypertension, for example, has been rigorously assessed by randomized trials that have defined pathogenic blood pressure levels and identified the methods of treatment that can improve outcome. ${ }^{8,9}$ Stringent evidence requirements for screening are important because of the potential for screening to lead to ambiguous results, unnecessary work-up, iatrogenic harm, and false reassurance. ${ }^{7,10}$ To this point, the US Preventive Services Task Force recommends against referral for $B R C A$ counseling and testing in asymptomatic women in the general population, based on a systematic review of potential benefits and harms, and recommends instead that family history be assessed to identify candidates for testing. ${ }^{11}$ Because there is no evidence on the outcomes of routine testing of the 56-gene panel in unselected patients, we currently lack the basis to establish a standard of practice; arguably, patients should be aware of the evidence limitations if they are offered this screening option.

In considering what evidence would justify this approach, the first issue is test performance, including the need to minimize false-positive and ambiguous findings. ${ }^{4}$ Positive and negative predictive value may differ when an assay is used in an unselected population versus in clinically based testing. ${ }^{12}$ Indeed, in cases in which the prior probability or prevalence of disease is low (as is the case for most conditions referenced in the ACMG recommendations), the positive predictive value will necessarily be lower than that in situations of high disease prevalence. ${ }^{12}$ Adequate predictions of test performance will require data from diverse populations; reliable estimates of the phenotypic spectrum for rare genotypes will require accumulation of substantial WGS/WES data.

Screening also requires resources for timely and appropriate follow-up (including a knowledgeable clinician workforce), and evidence that the screening process produces a net benefit at an acceptable cost, taking into account potential harms. ${ }^{713-18}$ With opportunistic screening, other demands on health providers' time must be considered, including the potential that the added screening may displace time for other interventions more relevant to the patient seeking care. ${ }^{19}$ Research is thus needed to assess test performance; methods for implementing genomic screening, including appropriate education and counseling measures; and outcomes.

\section{PATIENT CHOICE}

The new ACMG recommendations define results from the 56-gene panel as a "minimum list of incidental findings" to be generated without reference to patient preferences. ${ }^{1}$ This position is contrary to an ACMG policy statement issued in 2012 on clinical genomic sequencing that stated "patients should be given the option of not receiving certain or secondary findings." 3 The new policy raises serious ethical and legal concerns.

Competent adult patients have an established right to refuse medical interventions recommended by their health-care providers. This right is present even when medical interventions are immediately lifesaving, for example, in the case of a Jehovah's Witness who refuses a blood transfusion in the face of a likely fatal hemorrhage. Unless patients' decision-making capacity is impaired, or their refusal of therapy constitutes a threat to public health, their right to refuse is virtually unlimited. ${ }^{20}$ The 
ACMG recommendations propose, however, that any patient accepting WGS/WES for a clinical indication must also accept analysis of the 56 genes identified in the recommendations, even when avoiding this analysis would not impair the test's capacity to address the clinical question that prompted testing.

It may be that many patients undergoing WGS/WES would appreciate being offered the additional screening proposed in the ACMG recommendations, on the actionability grounds cited by the ACMG. However, choice matters. Patients may wish to decline the additional analysis on a number of grounds, including the limited evidence on test performance and outcomes. Concepts of shared decision making and respect for patient preferences argue for offering meaningful choices wherever possible, with appropriate information to allow patients to choose the best option for themselves. ${ }^{21}$ Furthermore, involving the patient in the decision-making process helps the patient to anticipate potential results and the clinical recommendations that follow if, for example, (in this case) testing of the 56 genes were chosen. With appropriate evidence development, the choices offered could include additional genes beyond those stipulated by ACMG recommendations, including tests for autosomal-recessive carrier states or pharmacogenomics panels. But patients should be free to decline any of these options.

If patients decline additional testing, it follows that the laboratory should not perform the additional analyses. In a clarification statement issued after the recommendations, the ACMG notes that "patients cannot opt out of the laboratory's reporting of incidental findings to the ordering clinician," but anticipates that "the provider and patient will participate in a shared decision-making process regarding the return of results."22 This ambiguous statement suggests that the anticipated shared decision making relates merely to how and when pathogenic findings might be disclosed, not whether they will be disclosed. Indeed, the ACMG clarification statement states that "not reporting ... would be unethical."22 Beyond the fundamental problem of depriving patients of choice, the additional costs involved in generating screening results from the 56 genes are a concern to both patients and the health-care system. These costs should not be generated if the patient does not wish to have the results.

An approach to practice that does not include the option to refuse additional genomic findings could lead to the unintended consequence of patients' refusing WGS/WES when it is indicated. As an example, when WES is employed to detect a mutation in a child with a suspected genetic disorder, testing of a parent-child trio is indicated. Under the ACMG recommendations, all three persons would be required to accept testing for the 56 genes, potentially leading a parent to be unwilling to participate. The requirement to return the specified additional genetic information might thus interfere with the optimal care of a patient, an inflexibility that would be ironic for a technology commonly presented as an integral element of "personalized medicine." Instead, robust debate is needed among all stakeholders to identify strategies for offering appropriate choices to patients and the research needed to assess those strategies.

\section{TESTING IN CHILDREN}

The ACMG's recommendations raise particular concerns related to testing in children. An important indication for WGS/WES is the work-up of individuals whose clinical presentation strongly suggests a genetic condition for which conventional testing approaches are either unavailable or have failed to generate an answer. Many of these individuals will be children. The recommendations state that the 56 genes should be assessed, and pathogenic variants reported, irrespective of the age of the patient. This is a surprising recommendation, which should be considered in the context of several other policy documents on pediatric genetic testing generated over the past two decades, including a recent joint statement from the ACMG and the American Academy of Pediatrics. ${ }^{23,24}$ The consensus recommends avoiding testing children for known familial mutations for adult-onset disease, on the premise that such testing is inconsistent with the best interests of the child and the child's future autonomy.

In calling for mandatory screening of all 56 genes in children receiving WGS/WES, the ACMG recommendations argue that the screening process could benefit parents or other family members. This rationale contrasts sharply with prevailing justifications of health care for children in that it proposes testing a child to benefit others. The ACMG recommendation statement makes it clear that the goal is to ensure the discovery of preventable disease risks, on the theory that providing those benefits to parents is in the child's interests. ${ }^{1,22}$ Although true incidental findings (discovered in the analysis required to address the presenting clinical question) should be offered to parents, intentional screening of a child aimed at putative parental benefit is different. Our society does not mandate that competent adults obtain medical information and/or treatment, even lifesaving treatment, just because they are parents. To argue that parents must consent to information that may indirectly benefit them is contrary to their right to refuse medical information and treatment. The rationale offered for childhood testing in the recommendations, coupled with mandatory return regardless of parental wishes, needs critical discussion and cannot be considered an appropriate basis for a standard of practice at the present time.

\section{MOVING TOWARD CONSENSUS}

The ACMG recommendations were motivated by the goal of standardizing and optimizing clinical genomic testing. Many well-intentioned clinicians, scientists, and other experts put considerable effort into developing the recommendations, and they represent a valuable starting point for deliberation. However, to date, this important conversation has not been broad enough. In addition, active efforts are needed to engage all stakeholders in discussion about the best steps forward. At a minimum, those stakeholders include public health and genetics professionals; clinicians from primary care, general pathology, and other specialties; policy makers; research and healthcare funders; bioethicists; and the health-care consumers for whom genomic medicine is being developed. An important 
part of this discussion should be about patient choice and how best to support it in the era of genomic testing.

Gathering more evidence is also essential. WGS/WES is already finding a place in clinical practice, as an expansion of existing genetic testing in certain defined clinical circumstances. This approach to practice innovation represents an accepted strategy, but efforts are needed to ensure that we learn as much as we can from early uses of the technology, through both observation of patient experience and clinical outcomes and controlled interventions designed to answer specific questions about the comparative effectiveness of WGS/WES versus other testing approaches.

Studies designed to assess genotype penetrance, effectiveness of medical follow-up, and personal and societal impact are particularly important for genome sequencing when the analysis is undertaken to achieve a screening objective. In this use, careful consideration must be given to potential harms, both to the individual tested and to the health-care system. These harms include adverse labeling of healthy people as "sick," unnecessary health-care expenditures, and iatrogenic complications., ${ }^{73-18}$ Because many of the conditions on the ACMG list are rare, consensus will be needed regarding the level of evidence that is sufficient to justify screening asymptomatic people.

Some relevant studies are already in process, funded by the National Human Genome Research Institute's Clinical Sequencing Exploratory Research. ${ }^{25}$ As part of the investigation of clinical uses of genome sequencing, the Clinical Sequencing Exploratory Research studies will evaluate the return of what it refers to as "unintended findings" and explore related normative and policy questions. Each of the Clinical Sequencing Exploratory Research studies defines unintended findings differently, and only a subset of these studies involve randomized trials evaluating the process of returning these findings, but they will provide valuable initial data to assess technical and clinical issues arising in evaluation and return of findings unrelated to the clinical purpose for testing. In addition to clinical sequencing studies, the Clinical Sequencing Exploratory Research consortium includes smaller studies funded by the National Human Genome Research Institute Ethical, Legal, and Social Implications research program that will provide additional data, insights, and normative analyses relevant to guideline development. Another study recently funded by the Ethical, Legal, and Social Implications program will design and pilot an assessment of a preventive genomic testing protocol for high-risk mutations, similar to those 56 identified in the ACMG recommendations, in a cohort of 1,000 adults. ${ }^{26}$ This study will provide additional social, behavioral, and policy-relevant data on the question of genomic screening.

Because evidence will accumulate over time, any near-term approaches to genome-sequencing practice should be provisional. Regular updating will be needed, with ongoing discussion of the implications of emerging evidence and clinical experience. This evidence will help stakeholders to assess the benefits and harms of opportunistic genomic screening such as the ACMG recommendations describe. As part of this effort, there is a need for clear processes for seeking patient consent, as well as a need for discussion of inclusion and exclusion criteria to identify candidate variants to be evaluated under different clinical scenarios, with systematic procedures to implement those criteria. The work of Goddard et al. ${ }^{27}$ sponsored by the Evaluation of Genomic Applications in Practice and Prevention working group represents an important step toward systematic evaluation.

Most important, the genetics community needs to ensure active debate of different uses of genome sequencing, different approaches to ensuring and supporting patient choice, and the ethical frameworks and evidence base needed to justify those uses. We need to invite other perspectives into the conversation and create forums that allow for respectful exchange of competing views. As an extraordinarily powerful and innovative technology, genome sequencing has the potential for both benefit and harm to patients. Joint and dedicated effort is needed to ensure positive and appropriate integration of genomics into clinical care.

\section{ACKNOWLEDGMENTS}

This work was supported in part by National Institutes of Health grants P5OHG003374 (W.B., G.P.J., B.W.), 1R21HG00612 (E.W.C, B.M.K., L.F.R.), P5OHG004488 (G.E.H.), 1R01HG006615 (I.A.H.), HG005491 (I.A.H.), U01HG006507 (G.P.J., W.B.), U01HG006375 (G.P.J.), R01HG005092 (W.R.U.), R01HG002213 (W.R.U.), and 1R01CA154517 (S.M.W.), Genome Canada/Genome Quebec (B.M.K.), and Robert Wood Johnson Foundation grant 69763 (S.M.W.).

Opinions expressed in this article are those of the authors and do not reflect the official position of the Centers for Disease Control and Prevention, the National Institutes of Health, or other funding agencies or institutions with which the authors are affiliated. All authors contributed substantively to this work and are listed alphabetically after the first author.

\section{DISCLOSURE}

The authors declare no conflict of interest.

\section{REFERENCES}

1. Green RC, Berg JS, Grody WW, et al. ACMG recommendations for reporting of incidental findings in clinical exome and genome sequencing. Genet Med 2013;15:565-574.

2. Jarvik G, Turner E, Robertson $P$, et al. Actionable, known pathogenic incidental findings in 1000 subjects' exomes. American College of Medical Genetics Annual Meeting, Phoenix, AZ, 21 March 2013.

3. American College of Medical Genetics and Genomics (ACMG). Points to consider in the clinical application of genomic sequencing. Genet Med 2012;14:759-761.

4. Evans JP, Berg JS, Olshan AF, Magnuson T, Rimer BK. We screen newborns, don't we?: realizing the promise of public health genomics. Genet Med 2013;15:332-334.

5. Ajioka RS, Kushner JP. Hereditary hemochromatosis. Semin Hematol 2002;39:235-241.

6. Begg CB. On the use of familial aggregation in population-based case probands for calculating penetrance. J Natl Cancer Inst 2002;94:1221-1226.

7. Wilson JMG, Jungner F. Principles and Practice of Screening for Disease. Public Health Papers No. 34, World Health Organization, Geneva, 1968.

8. Collins R, Peto R, MacMahon S, et al. Blood pressure, stroke, and coronary heart disease. Part 2, Short-term reductions in blood pressure: overview of randomised drug trials in their epidemiological context. Lancet 1990;335:827-838. 
9. Daskalopoulou SS, Khan NA, Quinn RR, et al.; Canadian Hypertension Education Program. The 2012 Canadian hypertension education program recommendations for the management of hypertension: blood pressure measurement, diagnosis, assessment of risk, and therapy. Can J Cardiol 2012;28:270-287.

10. Deyo RA. Cascade effects of medical technology. Annu Rev Public Health 2002:23:23-44.

11. US Preventive Services Task Force Draft Recommendation Statement. Risk assessment, genetic counseling, and genetic testing for BRCA-related cancer. http://www.uspreventiveservicestaskforce.org/draftrec.htm. Accessed 10 May 2013.

12. Zimmern RL. Issues concerning the evaluation and regulation of predictive genetic testing. J Community Genet 2012; e-pub ahead of print 8 July 2012

13. Burke W, Coughlin SS, Lee NC, Weed DL, Khoury MJ. Application of population screening principles to genetic screening for adult-onset conditions. Genet Test 2001;5:201-211.

14. Grosse SD, Rogowski WH, Ross LF, Cornel MC, Dondorp WJ, Khoury MJ. Population screening for genetic disorders in the $21^{\text {st }}$ century: evidence, economics, and ethics. Public Health Genomics 2010;13:106-115.

15. Harris R. Overview of screening: where we are and where we may be headed Epidemiol Rev 2011;33:1-6.

16. Rothstein MA. The case against precipitous, population-wide, whole genome sequencing. J Law Med Ethics 2012;40:682-689.

17. Viera AJ. Predisease: when does it make sense? Epidemiol Rev 2011;33: 122-134.

18. Welch G, Allen NA, Zagarins SE, Stamp KD, Bursell SE, Kedziora RJ. Comprehensive diabetes management program for poorly controlled Hispanic type 2 patients at a community health center. Diabetes Educ 2011;37: 680-688.

19. Getz L, Sigurdsson JA, Hetlevik I. Is opportunistic disease prevention in the consultation ethically justifiable? BMJ 2003;327:498-500.

20. Meisel A, Cerminara KL. The Right to Die: The Law on End-of-Life Decision Making, 3rd edn. Aspen: New York, 2004.

21. Spatz ES, Spertus JA. Shared decision making: a path toward improved patientcentered outcomes. Circ Cardiovasc Qual Outcomes. 2012;5(6):e75-e77.

22. American College of Medical Genetics and Genomics. Incidental findings in clinical genomics: a clarification. Genet Med 2013;15:664-666.

23. American Academy of Pediatrics Committee on Bioethics, Committee on Genetics and the American College of Medical Genetics and Genomics Social, Ethical and Legal Issues Committee. Policy statement: ethical and policy issues in genetic testing and screening of children. Pediatrics 2013; 131:620-622.

24. Ross LF, Saal HM, David KL, Anderson RR; American Academy of Pediatrics; American College of Medical Genetics and Genomics. Technical report: ethical and policy issues in genetic testing and screening of children. Genet Med 2013;15:234-245.

25. National Human Genome Research Institute. Clinical sequencing exploratory research. http://www.genome.gov/27546194. Accessed 10 May 2013.

26. University of North Carolina Center for Genomics and Society http://genomics. unc.edu/genomicsandsociety. Accessed 10 May 2013.

27. Goddard KA, Whitlock EP, Berg JS, et al. Description and pilot results from a novel method for evaluating return of incidental findings from nextgeneration sequencing technologies. Genet Med 2013; e-pub ahead of print 4 April 2013. 